\title{
Die schlecht heilende proximale Tibiafraktur - Infektion, Malunion, Delayed Union, Nonunion - was nun?
}

Benjamin Schoof, Tim-Nicolas Hissnauer, Till Orla Klatte, Karl-Heinz Frosch

\author{
Die Fraktur der proximalen Tibia mit und ohne Gelenkbeteiligung ist eine schwere \\ und komplikationsträchtige Verletzung. Sie stellt Patienten und Behandler aufgrund \\ der operativen Anforderungen und individuellen Behandlung vor eine herausfor- \\ dernde Aufgabe. Im folgenden Artikel werden Risiken und Komplikationen dar- \\ gestellt sowie mögliche Behandlungspfade aufgezeigt.
}

\section{Einleitung}

Die Tibia ist der am häufigsten gebrochene lange Röhrenknochen des Menschen. Zieht man alle Frakturen der Tibia heran, so betrifft jede 2. Fraktur die proximale Tibia [1]. Ursächlich sind zu je einem Drittel Sportverletzungen, Verkehrsunfälle und Stürze [2-4]. Bei jungen Patienten sind direkte Traumamechanismen im Sinne von Hochrasanztraumata oder Stürzen aus großer Höhe die häufigste Ursache. Beim alten Patienten stehen meist indirekte Mechanismen im Sinne von Niedrigenergietraumata in Verbindung mit osteoporotischem Knochenstatus im Vordergrund. Ermüdungsbrüche oder pathologische Frakturen sind selten.

Mit zunehmender Schwere der Fraktur - insbesondere im Vergleich zwischen unikondylärer und bikondylärer Beteiligung - steigt das Komplikationsrisiko und das klinische Outcome wird zunehmend reduziert [5-7]. Jeder 4. Patient zeigt eine im mittel- bzw. langfristigen Verlauf reduzierte Funktion oder fortschreitende Arthrose des Kniegelenks. Bei Auftreten eines periimplantären Infektes gilt dies für jeden 2. Patienten $[8,9]$.

Studien haben gezeigt, dass verzögerte Knochenheilung mit einem deutlich erhöhten Kostenaufwand einhergeht [10].

\section{Lokalisation und Biomechanik}

Die Frakturlokalisation an der proximalen Tibia (extraartikulär) stellt den Behandler vor biomechanische Schwierigkeiten. Zum einen zeigt sich ein meist kurzes, proximales Fragment mit Ansätzen ligamentärer und muskulärer Strukturen, deren Zugrichtung oft zu einer Rotation und Verkippung des Tibiakopfes führt. Durch die gegensätzliche Krafteinleitung ist das Dislokationsrisiko im Vergleich zu Frakturen anderer Tibiaabschnitte deutlich erhöht.
Das Tibiaplateau zeigt zur Tibiaschaftachse eine physiologische Retroposition und Retroversion (Slope) von 5-8 ${ }^{\circ}$. Im Fall einer Kompressions- oder Stauchungsbelastung wird hier die Abkippungstendenz des Tibiaplateaus durch die Krafteinleitung von M. quadriceps femoris (ventral, kranial) und M. soleus (dorsal, kaudal) zusätzlich verstärkt. Die ventralseitigen Zugkräfte sind im proximalen Bereich der Tibia am stärksten ausgeprägt und stellen in Kombination mit der dorsalen Abkippungstendenz eine hohe Dislokationsneigung und damit eine Herausforderung für die operative Versorgung dar [11].

\section{Klassifikation der Tibiakopffraktur}

Im europäischen Raum wird vorrangig die AO-Klassifikation (AO: Arbeitsgemeinschaft für Osteosynthesefragen) angewendet ( $\bullet$ Abb. 1) [12].

Frakturen der proximalen Tibia beinhalten somit die Typen 41A2 und A3 ohne Gelenkbeteiligung, die Typen 41A1, 41B und 41C mit Gelenkbeteiligung und die Typen 42 für diaphysäre Schaftfrakturen bei Lokalisation der Fraktur innerhalb der proximalen $2 / 5$ des Schaftes. Die gebräuchlichste Klassifikation ist die Schatzker-Klassifikation aus dem Jahr 1979 ( $\triangleright$ Abb. 2) [13].

Diese findet vor allem im amerikanischen Raum Anwendung. Zur Verbesserung der operativen Planung einer osteosynthetischen Versorgung von Tibiakopffrakturen wurde die 10-Segment-Klassifikation entwickelt, die ihr Hauptaugenmerk auf die Frakturlokalisation und intraoperative Einsehbarkeit des frakturierten Tibiaplateauanteils in Abhängigkeit vom operativen Zugangsweg legt $(\triangleright$ Abb. 3) [14].

Die Klassifikation eines begleitenden Weichteilschadens erfolgt nach Tscherne und Oestern $[15,16]$ die der offenen Frakturen nach Anderson und Gustilo [16]. 


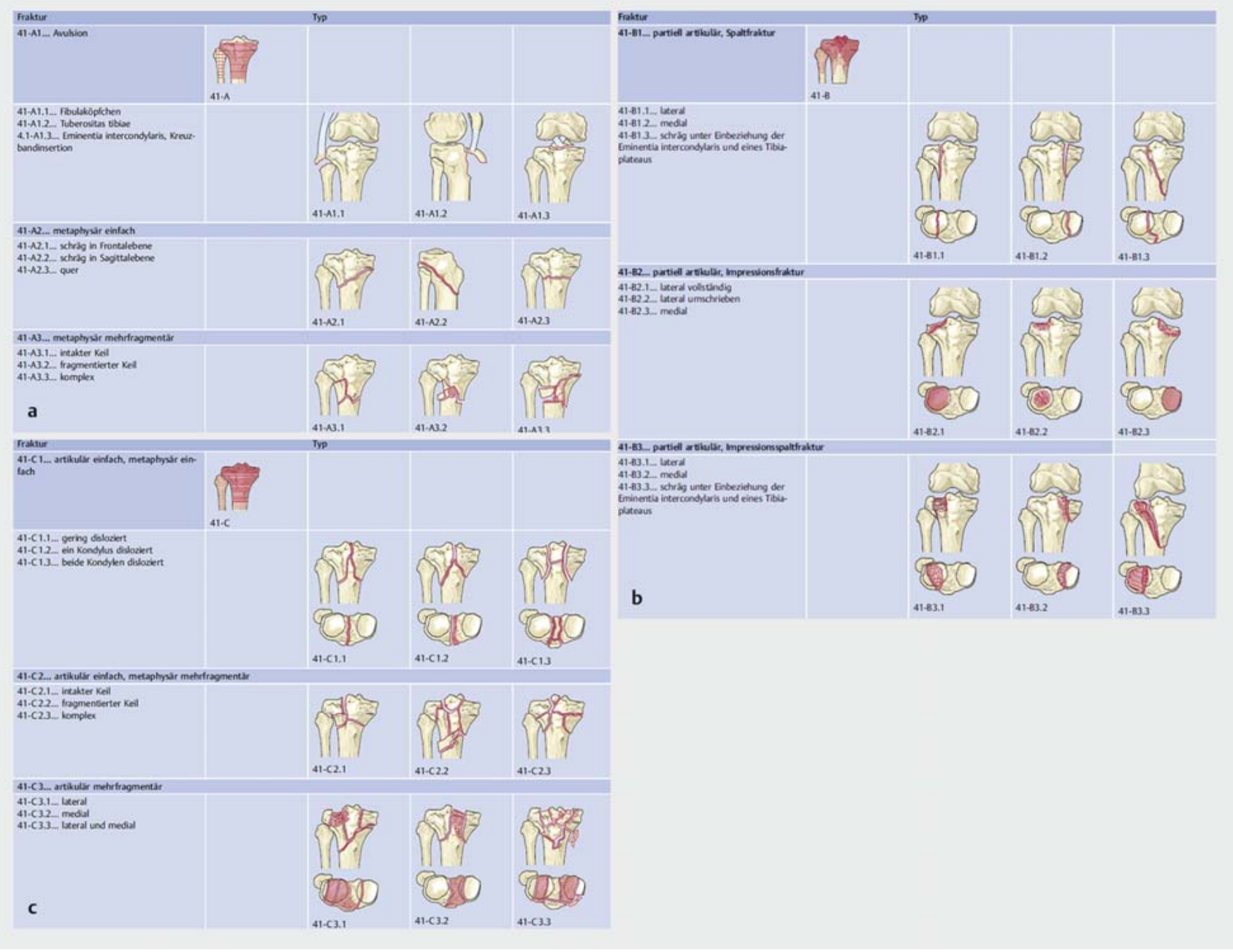

- Abb. 1 AO-Klassifikation. Quelle: Steinbrich W, Regazzoni P, Hrsg. Frakturen und Luxationen. Stuttgart: Thieme; 1999.

Merke

Essenziell für die Behandlung von Komplikationen ist das Verständnis der Ursache - bei der Infektion das Pathogen, bei der Malunion biomechanische Grundlagen und Korrekturmaßnahmen und bei der Pseudarthrose die zugrunde liegende Ursache.

\section{Infektion}

Die periimplantäre Infektion gehört zu einer der schwerwiegendsten Komplikationen und führt zu einem deutlich schlechteren Outcome $[9,10,19]$. Statistisch betragen die postoperativen Infektionsraten nach osteosynthetischer Frakturversorgung 1-5\% [20, 21]. Im Vergleich zu anderen Frakturen sind hier der Tibiakopf mit 14\% [7] und der proximale Tibiaschaft etwas häufiger betroffen [21 - 28]. Wenngleich die Ursache der hohen Infektionsrate nicht abschließend geklärt ist, scheinen beim Tibiakopf die Schwere der Fraktur mit bikondylärer Beteiligung (Schatzker Typ V) und begleitende Weichteilverletzungen mit einhergehenden multiplen operativen Eingriffen bzw. mehreren operativen Zugangswegen eine Erklärung zu sein. In der Gruppe der Frakturen der proximalen Tibia sind die offenen Frakturen etwas häufiger als geschlossene Frakturen betroffen. Dies betrifft nach gegenwärtiger Datenlage von 2-4\% für Frakturen 1.-2. Grades nach Gustilo und Anderson bis zu jeden 2. Patienten für Grad3-Frakturen [9,29]. Chronologisch lässt sich ein Infektgeschehen früh, verzögert oder spät einordnen. In Abhängigkeit von der Zeit treten einige Erreger gehäuft auf. $(\triangleright$ Tab. 1) [19].

Die Folgen einer periimplantären Infektion sind drastisch, so wird durchschnittlich jede periimplantäre Infektion im Bereich der proximalen Tibia ca. 5-mal revidiert [22]. In 0-5\% kommt es zur Ausbildung einer revisionsbedürftigen Gelenksteife bis hin zur Arthrodese [5-7].

\section{Behandlung}

Oberflächlicher Wundinfekt

Bei der Behandlung der periimplantären Infektion muss zwischen oberflächlichem und tiefem Wundinfekt unter- 


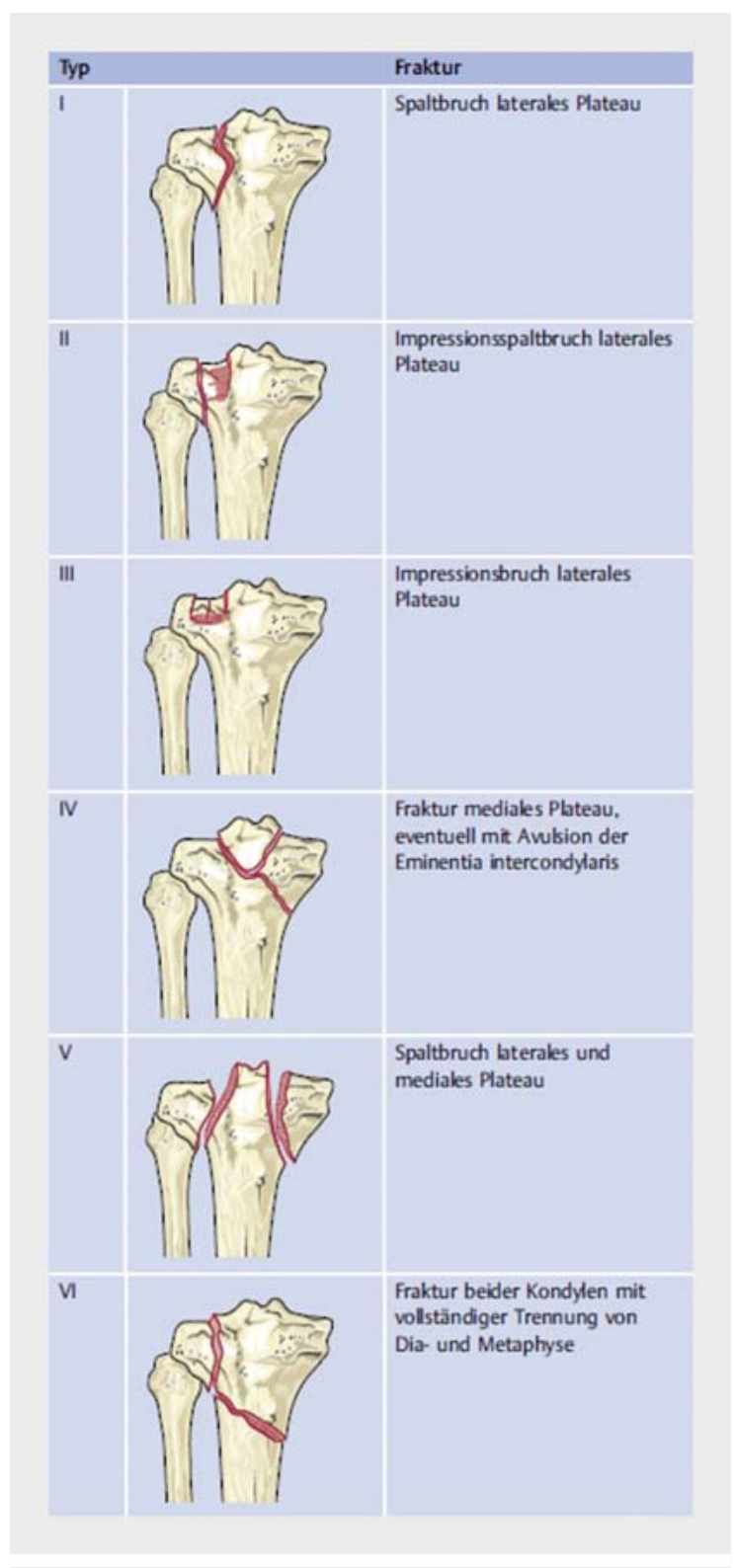

- Abb. 2 Schatzker-Klassifikation. Quelle: Steinbrich W, Regazzoni P, Hrsg. Frakturen und Luxationen. Stuttgart: Thieme; 1999.

schieden werden. Auch das Auftreten des Infektes im zeitlichen Kontext spielt eine große Rolle.

Im Falle des oberflächlichen Frühinfektes kann unmittelbar in der postoperativen Wundheilungsphase die frühzeitige Wundrevision mit lokalem Débridement und Spülung nach Entnahme von Gewebeproben zur mikrobiologischen Aufarbeitung zielführend sein. Es erfolgt die breite antibiotische Abdeckung und Anpassung der Antibiose nach Keimnachweis sowie vorliegendem Antibiogramm. Die Einlage von Antibiotikaträgern (Ketten, Schwämme) kann erwogen werden. Im eigenen Vorgehen wird jedoch
- Tab. 1 Chronologische Infekteinteilung [19].

\begin{tabular}{|c|c|c|}
\hline & Intervall & Keime \\
\hline Frühinfekt & 1.-14. Tag & $\begin{array}{l}\text { Staphylococcus aureus } \\
\text { gramnegative Aerobier } \\
\text { Staphylococcus pyogenes }\end{array}$ \\
\hline $\begin{array}{l}\text { verzögerter } \\
\text { Wundinfekt }\end{array}$ & 3.-10. Woche & $\begin{array}{l}\text { Staphylococcus aureus } \\
\text { koagulasenegative Staphylokokken }\end{array}$ \\
\hline Spätinfekt & > 10 Wochen & $\begin{array}{l}\text { Staphylococcus aureus } \\
\text { Staphylococcus epidermidis } \\
\text { Pseudomonas aeruginosa }\end{array}$ \\
\hline
\end{tabular}

insbesondere auf die Verwendung von Antibiotikaketten verzichtet. Da vor allem Biofilmbildner nach schneller Reduktion der freigesetzten Antibiotika auf der dann wirkungslosen Kette weitere Besiedelungsflächen finden. Die weitere Therapie richtet sich nach Wundstatus und laborchemischen Infektparametern (C-reaktives Protein und Leukozyten). Eine vollständige Materialentfernung ist indiziert, wenn die Infektion unter Materialerhalt nicht sanierbar ist.

Cave

Einliegende Ketten und Schwämme stellen nach Abgabe des antibiotischen Wirkstoffes Fremdkörper dar, die eine erneute Infektion begünstigen und daher entfernt werden sollten. Die Kettenglieder sind zu zählen und im OP-Bericht zu vermerken, damit eine vollständige Materialentfernung erfolgen kann.

\section{Tiefe Wundinfektion}

Die tiefe Wundinfektion tritt häufiger bei verzögerter und später Infektion auf. Ein früher postoperativer Beginn schließt jedoch eine tiefe Infektion niemals aus! Für die Behandlung der tiefen periimplantären Infektion ist ein ausgedehntes Débridement von infiziertem und nekrotischem Gewebe essenziell. Es sollten mindestens 5 mikrobiologische Gewebeproben aus unterschiedlichen Bereichen des Situs gewonnen werden [30]. Im Falle einer unzureichenden Weichteildeckung sollte frühzeitig die Weichteildeckung mit lokalen oder gestielten Muskellappen und Meshgraft angestrebt werden. Eine Vakuumbehandlung sollte, wenn überhaupt, nur kurzfristig erwogen werden. Insbesondere bei freiliegendem Knochen ist von einer Vakuumbehandlung abzuraten, sie stellt den absoluten Ausnahmefall dar.

Innerhalb der ersten 3 bis 4 Wochen nach primärem Eingriff kann versucht werden, das Osteosynthesematerial zu belassen. Später ist die vollständige Materialentfernung angeraten.

Postoperativ bzw. nach Entnahme der mikrobiologischen Proben wird wie beim Frühinfekt eine breitbandige anti- 


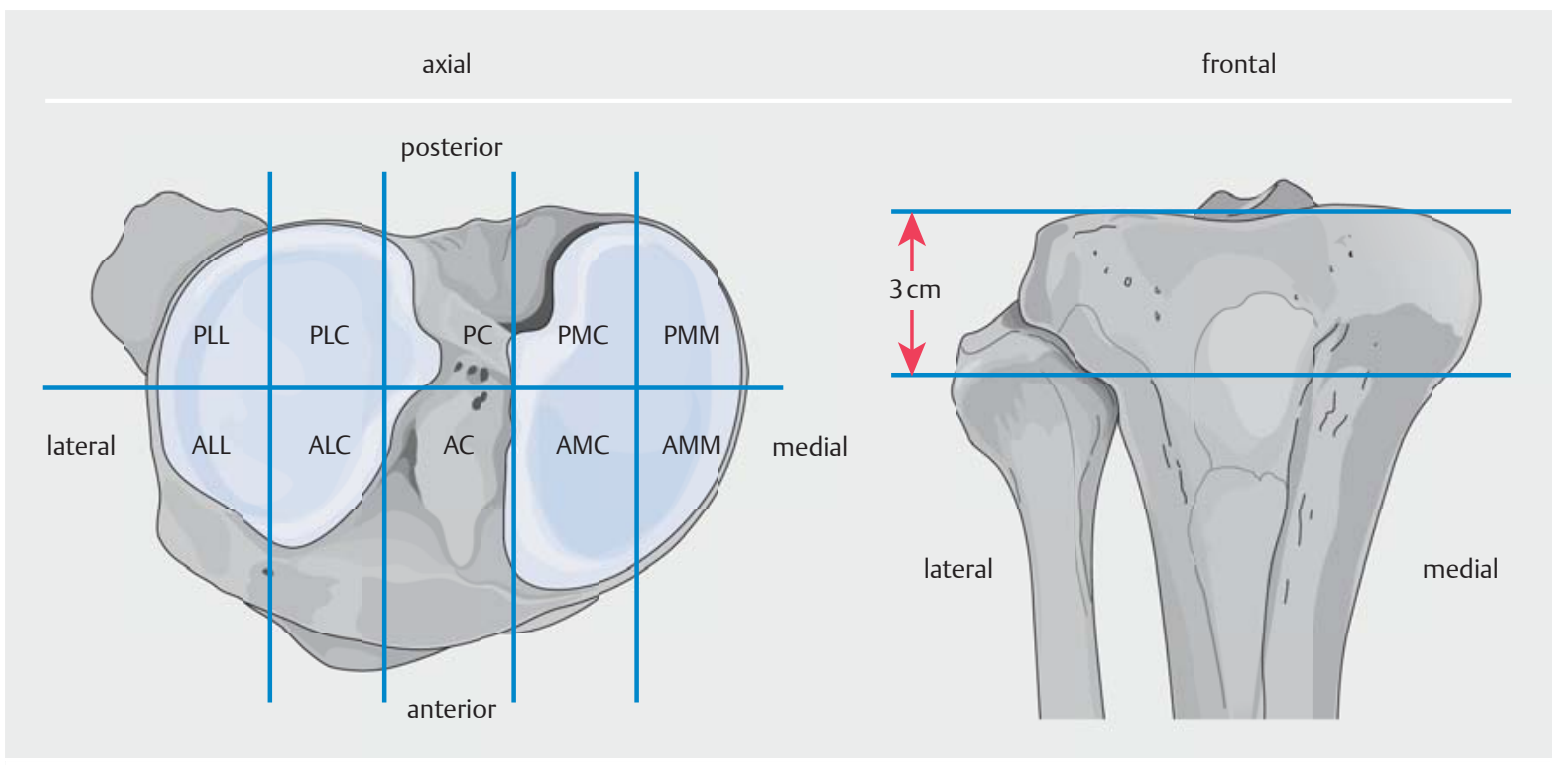

Abb. 3 10-Segment-Klassifikation. Aufteilung des Plateaus in 10 Segmente - jeweils 5 ventrale und 5 dorsale Segmente [14].

biotische Therapie begonnen. Diese muss nach Erhalt eines Resistogramms angepasst werden. Falls das einliegende Osteosynthesematerial entfernt werden muss, erfolgt die temporäre Fixierung der Fraktur mittels Fixateur externe. Hier ist auf einen ausreichenden Abstand der Pins zum infizierten Bereich zu achten. Gegebenenfalls ist dieser gelenkübergreifend anzulegen.

In Abhängigkeit vom Weichteilbefund werden im Intervall Wundrevisionen mit erneuter Sicherung mikrobiologischer Proben durchgeführt. Im Speziellen muss hier auf Keimwechsel und Änderung der Resistenzlage geachtet werden. Durch radikales Débridement sollten mehrfache Eingriffe vermieden werden.

Merke

Oberstes Ziel ist die Sanierung des Infektes durch frühzeitige operative Intervention und radikales Débridement. Der Keimnachweis für eine resistenzgerechte Antibiose ist notwendig! Die präoperative antibiotische Therapie sollte vermieden werden, sofern für den Zustand des Patienten vertretbar!

\section{Malunion}

Zu einer Malunion (= Fehlstellung) kommt es nach mangelnder Reposition, Materialversagen, Fragmentdislokation oder Knochentransplantatnekrose. Bikondyläre Frakturen sind häufiger betroffen. Schlechte Knochenqualität und hohes Alter sind Risikofaktoren für eine Malunion [7, 31].
Malunion kann definiert werden als Repositionsverlust mit einer Gelenkstufe > 3 mm oder einer Varus-/Valgusfehlstellung $>5^{\circ}$ [31]. Hauptaugenmerk der Behandlung muss die Wiederherstellung der Beinachsen, Gelenklinie und Stabilität sein. Zur präoperativen Planung nach fehlverheilter Fraktur sind eine Ganzbeinaufnahme, seitliche Slope-Aufnahme-CT-Diagnostik und ggf. ein MRT wegweisend für die Behandlung. Bei ausgedehnten Knochendefekten bei osteoporotischem Knochenstatus des älteren Patienten ist die Implantation einer Knieendoprothese zu erwägen [11]. Da Studien gezeigt haben, dass Patienten selbst nach unzureichender Reposition in Ausnahmefällen gute funktionelle Ergebnisse bieten können [32] und die Tibiakopfoberfläche residuelle Inkongruenzen ohne Ausbildung von Degenerationen aufweisen kann [23], sind operative Revisionseingriffe immer auch von den Beschwerden des Patienten abhängig zu machen.

\section{Ziel der Revision ist die Verbesserung des Schmerz- levels und der Gelenkfunktion}

Ist die einzeitige Korrekturosteotomie bei ausgeprägten Achsfehlstellungen und eventuellen Längendifferenzen nicht einzeitig machbar, können externe Fixateursysteme zum Einsatz kommen, die eine schrittweise Korrektur und damit eine Wiederherstellung der physiologischen Achsenverhältnisse ermöglichen. Sie bieten die Möglichkeit, eine komplexe Fehlstellung mit täglichen Feinjustierungen zu einer anatomischen Beinachse und -länge wiederherzustellen [26, 27]. 
Für alle posttraumatischen Beinachsendeformitäten gilt, dass einem Revisionseingriff eine umfassende Planung der Korrektur unter Einbeziehung der Fehlstellung in koronarer und sagittaler Ebene vorausgehen sollte.

\section{Behandlung}

Im Falle einer sekundären Fehlstellung steht die Achskorrektur gemeinsam mit der knöchernen Durchbauung im Vordergrund. Im Falle der Beinlängendifferenz ist diese ebenfalls im Rahmen der Behandlung adressierbar.

Bei posttraumatischer Achsfehlstellung mit noch einliegendem Material sollte bei entsprechendem Verdacht weiteren operativen Prozeduren die vollständige Materialentfernung und eine mikrobiologische Probenentnahme vorausgehen, um eine infektiöse Genese auszuschließen.

Um ein zufriedenstellendes Ergebnis herstellen zu können, bedarf es hier einer intensiven präoperativen Planung. So ist die Osteotomielokalisation entscheidend für das Therapieergebnis. In den meisten Fällen ist es möglich, eine posttraumatische Valgusfehlstellung mit einer proximalen öffnenden Tibiaosteomie zu korrigieren [34]. Bei ausgeprägten Varusfehlstellungen ist eine valgisierende distale Femurosteotomie oder eine öffnende hohe tibiale Osteotomie möglich. In beiden Fällen ist eine Kombination mit intraartikulären Umstellungsosteotomien möglich, um eine kongruente Gelenklinie zu erreichen [35]. Ebenfalls muss bei der Auswahl der richtigen Korrekturosteotomie die Auswirkung auf die Beinlänge bedacht werden. So hat ein öffnendes Osteotomieverfahren einen größeren Einfluss auf die Beinverlängerung als ein schließendes Verfahren auf eine Beinlängenverkürzung [36].

Ist eine achsgerechte Stellung des Unterschenkels nicht sofort zu erzielen, muss die Beinachse und -länge schrittweise angeglichen werden. Einschränkend sind an dieser Stelle der Weichteilmantel und die Zugbelastung auf Nerven.

\section{FALLBEISPIEL}

Malunion - extraartikulär ( $\triangleright$ Abb. 4 a-j). 44-jährige Patientin mit Z.n. Überrolltrauma und nun posttraumatisch kombinierter Antetorsions- $\left(35^{\circ}\right)$ und Varusdeformität $\left(50^{\circ}\right)$ links nach (Unterschenkel-)Fraktur des proximalen Tibiadrittels und desolater Weichteilsituation.

\section{Delayed Union/Nonunion}

Die Raten von Knochenheilungsstörungen mit verzögerter (delayed union) oder ausbleibender (nonunion) Knochenheilung steigen mit der Schwere der Fraktur. Sie stellen mit ca. 4\% der Komplikationen nach schweren Tibiakopffrakturen [7] eine seltene, aber aufgrund der weitrei-

\section{INFOBOX - KALLUSDISTRAKTION}

Durch Distraktion auf den Osteotomiespalt wird der sich bildende Kallus auseinandergezogen und damit der Defekt bzw. die auszugleichende Länge überbrückt.

Hierzu werden nach einer anfänglichen Latenzphase in 1-mm-Schritten entsprechend einem IT-gestützten Korrekturplan täglich Anpassungen des Fixateurs durchgeführt ( $\vee$ Abb.4e-h). Eine mögliche Beinlängendifferenz kann hier mit ausgeglichen werden. Sind Strecke und Defektzone zu groß, kommt für eine Überbrückung ein sog. Segmenttransport infrage. Bei diesem wird durch eine 2. Osteotomie eine iatrogene Stückfraktur gebildet. Dieses Segment wird nun mittels der verstellbaren Fixateurstreben durch tägliche Justierungen durch die zu überbrückende Defektstrecke transportiert. Dabei zieht das Segment eine Kallusblase hinter sich her, von wo aus die Defektstrecke verknöchert. Moderne intramedulläre Devices haben - ebenfalls IT-gestützt - die Behandlung von Fehlstellungen und Beinlängendifferenzen der Röhrenknochen revolutioniert und die Nutzung von externen Fixateuren reduziert. Prinzipiell ist es möglich, die Osteotomieareale im Fixateur ausheilen zu lassen. Dies ist bei kritischen Weichteilsituationen ggf. notwendig. Favorisierte Versorgung am Tibiakopf sollte eine winkelstabile Plattenosteosynthese mit Anlagerung von autologem oder allogenem Knochenmaterial sein. Im Falle einer Gelenkflächenbeteiligung im Sinne eines sekundären Einbruches mit Veränderung von Gelenkachse und Slope besteht das Risiko einer Pseudoinstabilität im Kniegelenk. Einhergehend mit einem Instabilitätsgefühl kommt es zur Ausbildung einer sekundären Fehlstellung der Beinachse im Kniegelenk ( $\triangleright$ Abb. 5d).

\section{FALLBEISPIEL}

Malunion - artikulär ( $\triangleright$ Abb. 5a-f). 21-jährige Patientin nach Sturz beim Skifahren mit Grenzwertindikation zur intraartikulären öffnenden Osteotomie bei arthroskopischem Nachweis eines intakten lateralen Meniskus und jungem Alter.

chenden Folgen eine nicht zu unterschätzende Entität dar.

Gründe für die ausbleibende Knochenheilung sind ausgedehnte Trümmerzonen, ungenügende osteosynthetische Stabilität ( $\bullet$ Abb. 4 a-f), Nekrose des allogenen oder autologen Knochentransplantats, mechanisches Implantatversagen, Infektion oder die Kombination mehrerer genannter Komplikationen. Infrage kommen außerdem 

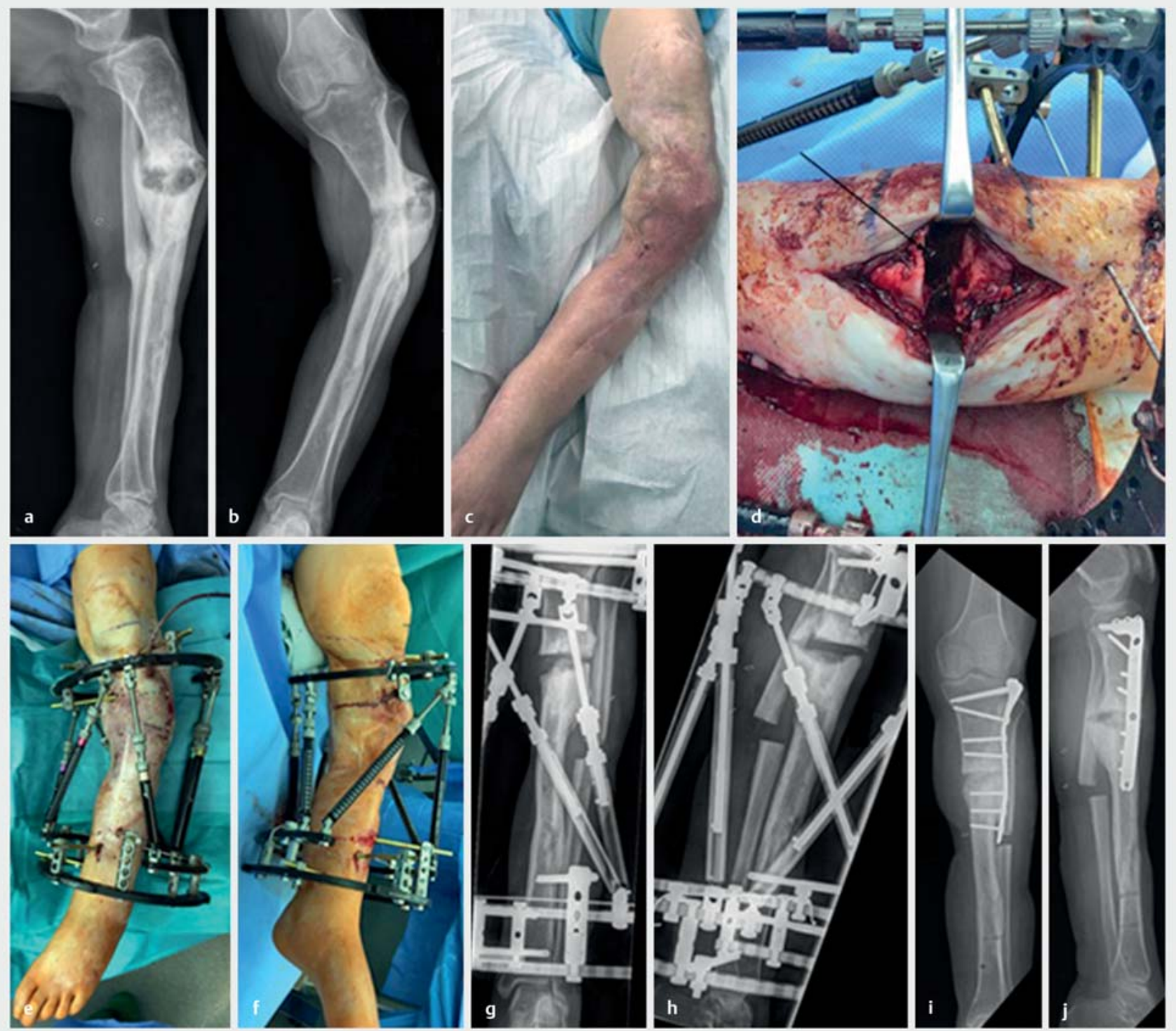

- Abb. 4 a-c Präoperative Antetorsions- und Varusdeformität. d Tibiale Pseudarthrosenresektionszone ( $\rightarrow$ ). e, $f$ Postoperativ nach Ringfixateuranlage und teilweiser Achskorrektur. g, h Postoperative Röntgenkontrolle im 10-wöchigen Ausgradungsintervall im TSF-Fixateur. i, j Röntgenkontrolle nach definitiver plattenosteosynthetischer Versorgung und Defektauffüllung mittels autologer Spongiosa.

in den Frakturspalt eingeschlagene Weichteile, lokale Durchblutungsstörungen, Diabetes mellitus und Gefäßerkrankungen wie eine pAVK.

\section{FALLBEISPIEL}

55-jähriger Patient mit AO-41A-Fraktur und lateraler Plattenosteosynthese (a, b) und mit Pseudarthrose nach 6 Monaten (c) und Re-Do mit ergänzender Plattenosteosynthese und allogener Spongiosaplastik (d, e) mit Heilungsbild nach 10 Wochen $(\mathbf{f})(\triangleright$ Abb. 6 a-f).
Radiologisch unterschieden werden hypertrophe, atrophe und synoviale Pseudarthrosen [37].

Weiterhin können Pseudarthrosen nach ihrer Stabilität als stabil, teilweise stabil und instabil eingeteilt werden [38, 39]. Steife hypertrophe Pseudarthrosen haben eine Angulation bis zu $5^{\circ}$ in einer Ebene, teilweise stabile hypertrophe Pseudarthrosen zwischen 5 und $20^{\circ}$ in einer Ebene und instabile hypertrophe Pseudarthrosen $>20^{\circ}$ multiplanar. Dabei haben steife Pseudarthrosen Bindegewebsknorpel, partiell stabile Pseudarthrosen straffes fibröses Bindegewebe und instabile Pseudarthrosen loses Bindegewebe oder Synovialgewebe im Frakturspalt. 


\section{INFOBOX}

Pseudoinstabilität - klinische und subjektive posttraumatische ligamentäre Instabilität bei intaktem Bandapparat, die durch Absenkung der Gelenkfläche und damit gesehen einer relativen „Überlänge“ der intakten Bänder einhergeht!

Nach Materialentfernung und arthroskopischer Sichtung der Knorpelverhältnisse intraartikulär ist dann entweder die Gelenkfläche mittels intraartikulärer Osteotomie zu rekonstruieren und/oder ergänzend eine Umstellungsosteotomie zur Beinachsenkorrektur durchzuführen ( $\triangleright$ Abb. 5 e). Zusätzlich muss eine ligamentäre Stabilisierung im Kniegelenk erwogen werden. Eine Rekonstruktion des Tibiaplateaus erfolgt über eine intraartikuläre Osteotomie, Unterfütterung mittels autologem oder allogenem Knochen und Stabilisierung mittels winkelstabiler Plattenosteosynthese.

Ligamentäre Instabilitäten werden durch diese Verfahren meist kompensiert. Ist dies nicht der Fall, kann in einem ein- oder zweizeitigen Verfahren eine Bandplastik zur Stabilisierung zusätzlich erfolgen.

Die Behandlung von Pseudarthrosen hängt von der zugrunde liegenden Ursache ab, stellt aber in jedem Falle eine anspruchsvolle individuelle Behandlung dar. Sowohl interne als auch externe Osteosyntheseverfahren kommen meist unter Zuhilfenahme von Spenderknochen (autolog/homolog) und Knochenersatzstoffen zum Einsatz.

\section{Merke}

Nach der aktuellen Definition der ESTROT (European Society of Tissue Regeneration in Orthopedics and Traumatology) ist eine Pseudarthrose eine Fraktur ohne Heilungstendenz - unabhängig von ihrer Behandlungsdauer! In Deutschland ist eine ausbleibende Heilung von 6 Monaten beweisend für eine Pseudarthrose [40].

\section{Behandlung Delayed Union/Nonunion}

Während die Behandlung der Pseudarthrose meist operativ erfolgt, besteht im Fall einer verzögerten Knochenheilung oft die Möglichkeit einer zunächst abwartenden konservativen Behandlung.

Im Falle einer Marknagelosteosynthese kommt gelegentlich eine Dynamisierung des Osteosynthesenagels durch Entfernung der Verriegelungsbolzen in Betracht. Dies kann, je nach Lokalisation, auch in regionaler Anästhesie erfolgen. Zwingend ist darauf zu achten, dass das dynamisierte Ende des Marknagels ausreichend Strecke zur Kompression aufweist. Durch die Dynamisierung des Marknagels unter gleichzeitiger Belastung wird der Frakturspalt komprimiert und eine Kallusreifung mit folgen-

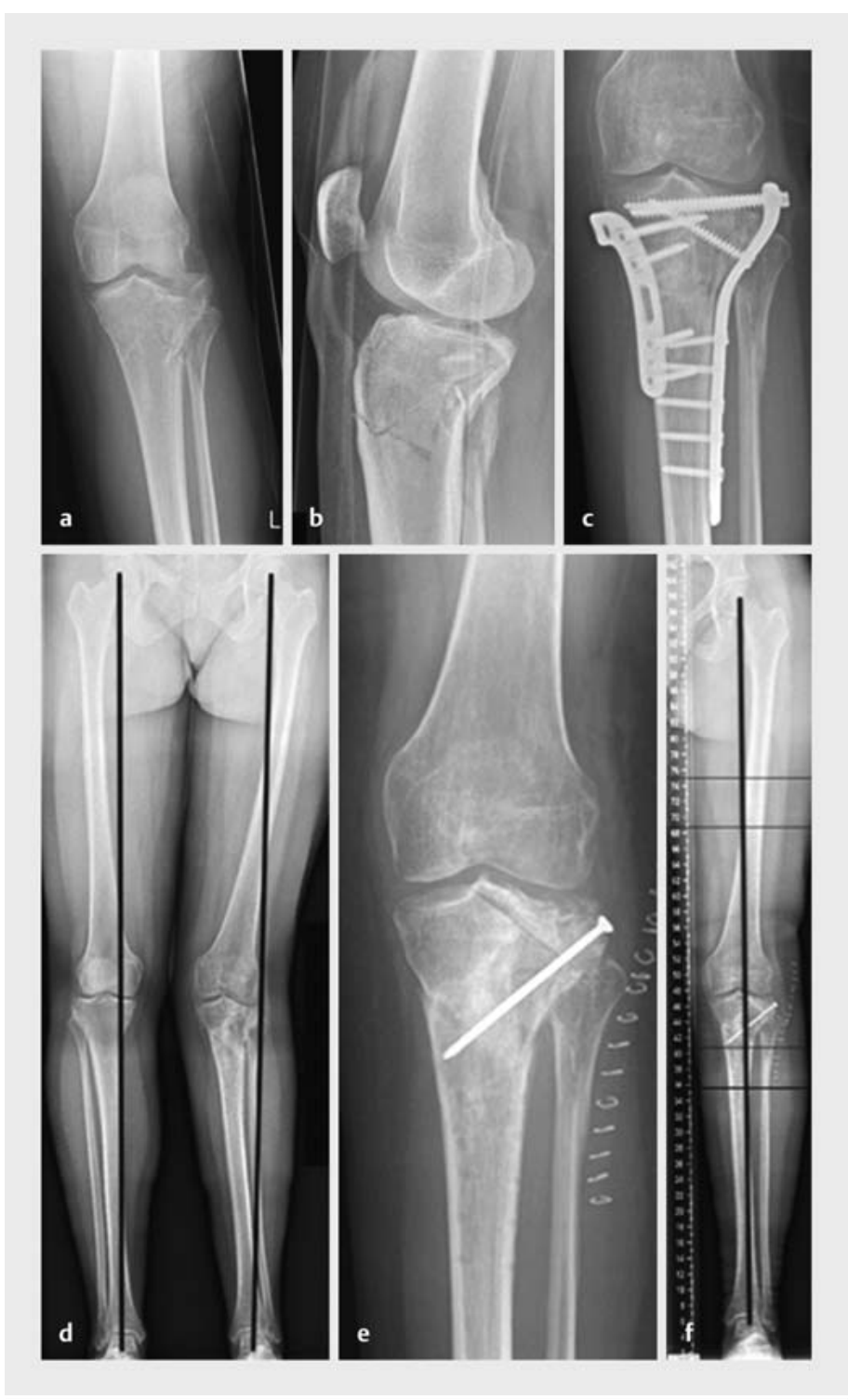

- Abb. 5 a, b Bikondyläre Tibiakopffraktur Schatzker VI; AO 41C2.1. c Röntgenkontrolle nach Plattenosteosynthese. d Ganzbeinstandaufnahme mit Mikulicz-Linie nach Materialentfernung und mikrobiologischer Probenentnahme. e Postoperative Röntgenkontrolle nach intraartikulärer lateral öffnender Osteotomie mit allogenem Spongiosatransplantat. f Postoperatives Ganzbeinstandbild mit korrigierter Tragachse.

der knöcherner Durchbauung induziert. Diese Methode kommt in erster Linie für schlecht heilende Querfrakturen im Schaftbereich infrage. Zu beachten ist, dass bei hypertrophen Pseudarthrosen meist eine gewisse mechanische Instabilität (z. B. zu dünner Marknagel) die Ursache sein kann. Durch Entfernung der Verriegelungsbolzen kann in solchen Fällen die Instabilität noch weiter begünstigt werden, sodass keine Heilung erfolgen wird! Dies ist präoperativ genau zu evaluieren! Auch ist eine starke Verkürzung der Extremität durch Entfernung der Verriegelungsbolzen zu vermeiden! 

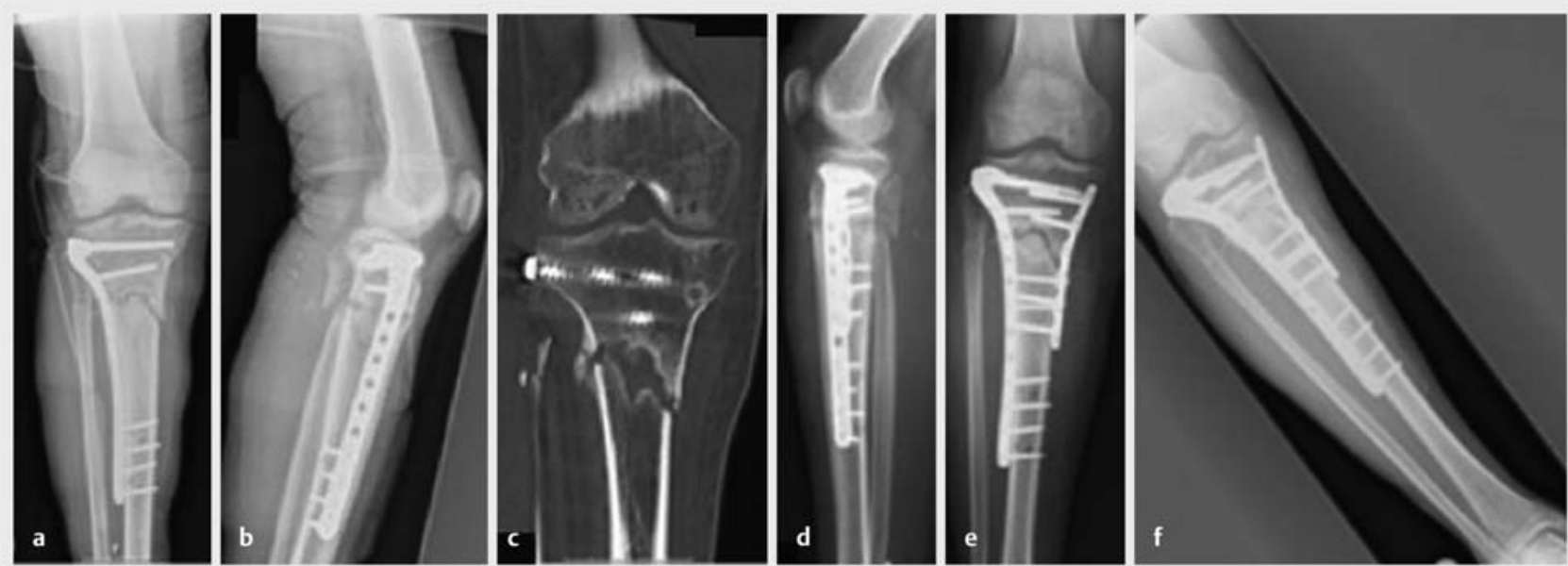

Abb. 6 Mangelnde Stabilität der Osteosynthese. Unicondyäre Plattenosteosynthese mit Pseudarthrosenbildung und Ausheilung nach Re-Do mit Spongiosaauffüllung und bilateraler Plattenosteosynthese.
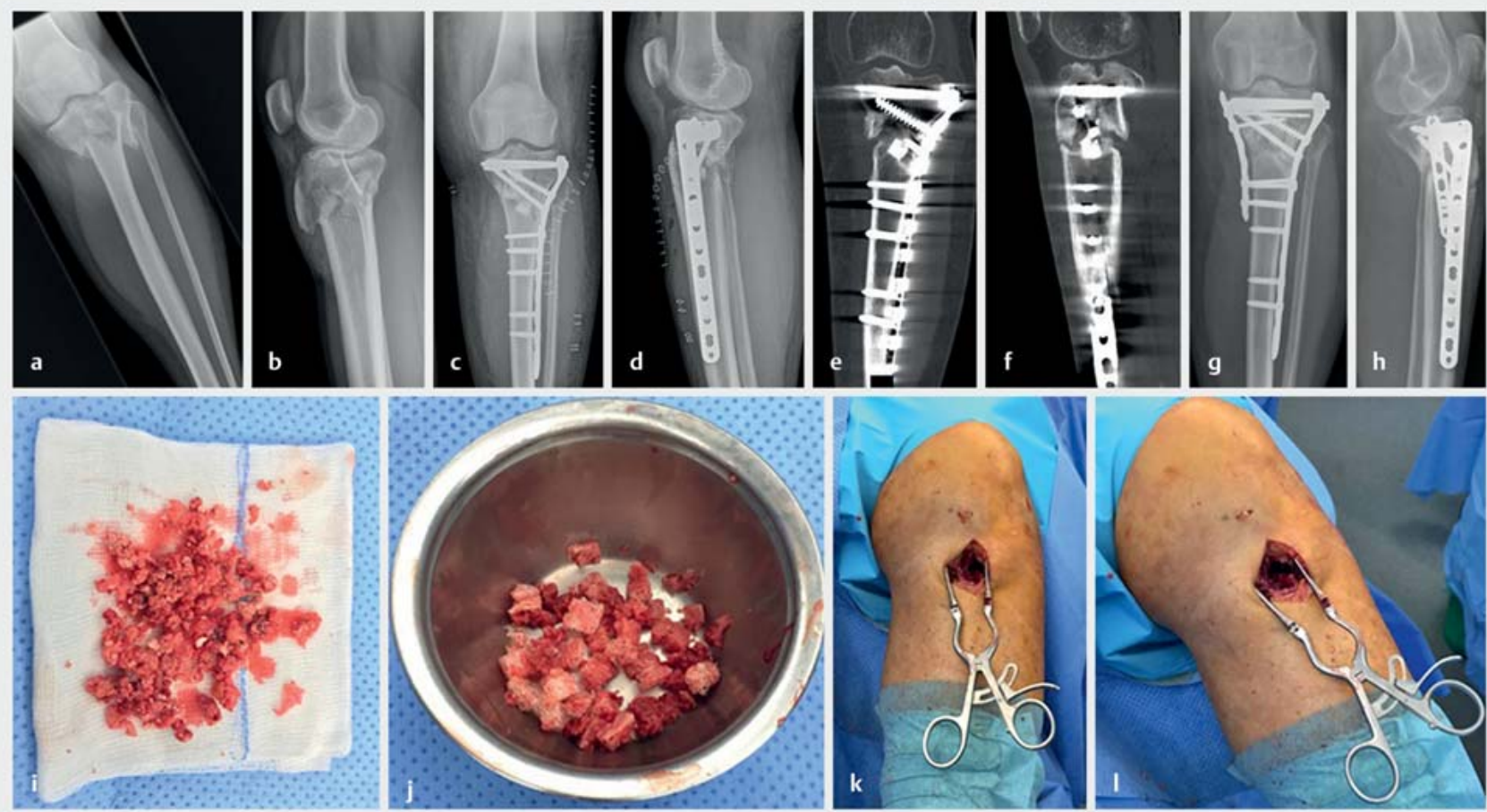

- Abb. 7 a, b Präoperative Röntgenbilder. c, d Röntgenbilder nach unilateraler winkelstabiler Plattenosteosynthese und Knochenersatzmaterial. e, $\mathbf{f}$ CT des Tibiakopfes mit Nachweis einer Pseudarthrose 6 Monate postoperativ. g, h Röntgenkontrolle nach Revision des Tibiakopfes nach Pseudarthrosenresektion, allogener Spongiosaplastik und medialer Plattenosteosynthese. i Pseudarthrosenresektat mit Knochenersatzmaterial („,Katzenstreu“). j Präparierte allogene Spongiosachips. k, I Anteromedialer Zugang zum Tibiakopf mit zentralem Knochendefekt der Pseudarthrose.

\section{FALLBEISPIEL}

Nonunion

53-jährige Patientin mit Pseudarthrose 1 Jahr nach eingestauchter Tibiakopffraktur AO 41C3.3 im Rahmen eines Skiunfalls ( $\triangleright$ Abb. $7 a-I)$.
Eine alternative Behandlungsmethode stellt die ultraschallgetriggerte Knocheninduktion dar. Hier wird von extern durch Aufsetzen eines Schallkopfes mit niedrig dosiertem gepulstem Ultraschall eine Zellkaskade in Gang gesetzt, die in der Lage sein kann, die Knochenheilung zu induzieren und zu beschleunigen. Die Datenlage hierzu ist jedoch begrenzt und kontrovers. So zeigen einige 
Studien eine Begünstigung der Knochenheilung, andere jedoch keine positiven Effekte auf Knochenheilung und Schmerzreduktion.

Die chirurgische Revision ist bei der atrophen und synovialen Pseudarthrose i.d.R. indiziert. Zunächst sollte der Ausschluss einer Infektion erfolgen und einliegendes Osteosynthesematerial entfernt werden. Bei zweizeitigem Vorgehen einer ausgeprägten Instabilität erfolgt die Anlage eines ggf. gelenkübergreifenden externen Fixateurs. Es folgt in einem nächsten Schritt die Pseudarthrosenresektion mit anschließender autologer oder allogener Spongiosaplastik und winkelstabiler Plattenosteosynthese ( $\bullet$ Abb. $\mathbf{7 g}-\mathbf{I})$. Auch hier ist im Bedarfsfall wie bei der Malunion die Anwendung eines Ringfixateurs zur Kallusdistraktion oder Achskorrektur möglich. Bei größeren Defekten kann auch das Masquelet-Verfahren erfolgreich sein. Die korrekte Achsenstellung sollte dabei beachtet werden.

\section{Cave}

Nach Pseudarthrosenresektion fehlt häufig eine knöcherne Referenz für die korrekte Achsenausrichtung! Auch die Torsion kann fehlerhaft sein und sollte präoperativ genau evaluiert werden!

\section{Chirurgische Alternativen}

Sind Knochenqualität, Gelenkkongruität oder Weichteilmantel (Haut, Streckapparat) irreparabel geschädigt, sind Knieprothese, Arthrodese oder Amputation letzte chirurgische Auswege für die Behandlung. Gelenkerhaltende Maßnahmen sind immer zu favorisieren. Eine endoprothetische Versorgung ist nur möglich im Falle der vollständigen Infektfreiheit. Die Implantation einer Endoprothese nach Tibiakopffraktur ist mit erhöhten Komplikationsraten im Vergleich mit der primären Endoprothetik verbunden. Materialentfernung und Implantation der Endoprothese sollten am Kniegelenk deshalb zweizeitig erfolgen! Die Amputation stellt in der Kette der Maßnahmen die letzte therapeutische Option dar.

\section{Fazit}

Die „schlecht heilende Fraktur der proximalen Tibia“ stellt eine ernsthafte Komplikation dar und kann weitreichende Folgen für die Funktion, die Leistungsfähigkeit und das Sozialleben des betroffenen Patienten haben. Diagnostik und Therapie können den Arzt vor große Herausforderungen stellen und setzen viel Erfahrung voraus. Eine exakte klinische und radiologische Analyse der Pathologie sowie ein genau geplantes chirurgisches Vorgehen sind Grundlage für ein gutes Outcome. Grundsätzlich gilt es auch, eine Infektion auszuschließen oder bei positivem Keimnachweis ein frühzeitiges und radikales chirurgisches Therapieregime einzuleiten.

\section{Interessenkonflikt}

Die Autoren geben an, dass kein Interessenkonflikt besteht.

Autorinnen/Autoren

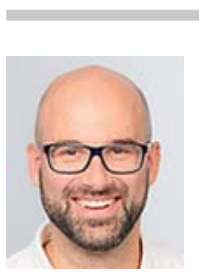

\section{Benjamin Schoof}

Dr. med., Wissenschaftlicher Mitarbeiter, Facharzt für Orthopädie und Unfallchirurgie, Klinik für Unfall-Hand- und Wiederherstellungschirurgie, Universitätsklinikum Hamburg-Eppendorf

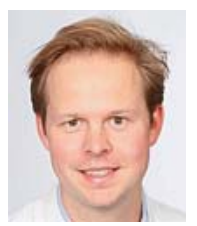

\section{Tim-Nicolas Hissnauer}

Dr. med., Wissenschaftlicher Mitarbeiter, Facharzt für Orthopädie und Unfallchirurgie, Klinik für Unfall-Hand- und Wiederherstellungschirurgie, Universitätsklinikum Hamburg-Eppendorf

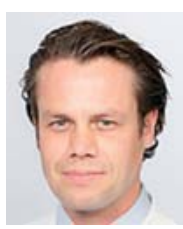

\section{Till Orla Klatte}

PD Dr. med., Oberarzt, Facharzt für Orthopädie und Unfallchirurgie, Spezielle Unfallchirurgie, Klinik für Unfall-Hand- und Wiederherstellungschirurgie, Universitätsklinikum HamburgEppendorf

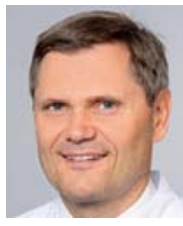

\section{Karl-Heinz Frosch}

Prof. Dr. med., Klinikdirektor, Facharzt für Orthopädie und Unfallchirurgie, Facharzt für Allgemeinchirurgie, Spezielle Unfallchirurgie, Klinik für Unfall-Hand- und Wiederherstellungschirurgie, Universitätsklinikum HamburgEppendorf

\section{Korrespondenzadresse}

\section{Prof. Dr. Karl-Heinz Frosch}

Klinik für Unfall-, Hand- und Wiederherstellungschirurgie

Universitätsklinikum Hamburg-Eppendorf

Martinistraße 52

20246 Hamburg

Tel.: 040/74 10-53459

Fax: 040/74 10-54569

unfallchirurgie@uke.de

\section{Literatur}

[1] Wennergren D, Bergdahl C, Ekelund J et al. Epidemiology and incidence of tibia fractures in the Swedish Fracture Register. Injury 2018; 49: 2068-2074

[2] Kraus TM, Martetschläger F, Müller D et al. Return to sports activity after tibial plateau fractures: 89 cases with minimum 24-month follow-up. Am J Sports Med 2012; 40: 2845-2852. doi:10.1177/0363546512462564

[3] Märdian S, Schwabe P, Schaser K-D. Tibiaschaftfrakturen. Z Orthop Unfall 2015; 153: 99-119

[4] Meisinger C, Wildner M, Stieber ] et al. [Epidemiology of limb fractures]. Orthopade 2002; 31: 92-99 
[5] Rademakers MV, Kerkhoffs GM, Sierevelt IN et al. Operative treatment of 109 tibial plateau fractures: five- to 27-year follow-up results. J Orthop Trauma 2007; 21: 5-10

[6] Barei DP, Nork SE, Mills WJ et al. Complications associated with internal fixation of high-energy bicondylar tibial plateau fractures utilizing a two-incision technique. J Orthop Trauma 2004; 18: 649-657

[7] Morris BJ, Unger RZ, Archer KR et al. Risk factors of infection after ORIF of bicondylar tibial plateau fractures. J Orthop Trauma 2013; 27: e196-e200

[8] Biggi F, Di Fabio S, D'Antimo C et al. Tibial plateau fractures: internal fixation with locking plates and the MIPO technique. Injury 2010; 41: 1178-1182

[9] Castillo RC, Bosse MJ, MacKenzie EJ et al. Impact of smoking on fracture healing and risk of complications in limb-threatening open tibia fractures. J Orthop Trauma 2005; 19: 151-157

[10] Henkelmann R, Frosch K-H, Glaab R et al. Infection following fractures of the proximal tibia - a systematic review of incidence and outcome. BMC Musculoskelet Disord 2017; 18: 481

[11] Heckman JD, Sarasohn-Kahn J. The economics of treating tibia fractures. The cost of delayed unions. Bull Hosp Jt Dis 1997; 56: 63-72

[12] Meinberg EG, Agel J, Roberts CS et al. Fracture and Dislocation Classification Compendium-2018. J Orthop Trauma 2018; 32 (Suppl. 1): S1-S170. doi:10.1097/BOT.0000000000001063

[13] Schatzker J, McBroom R, Bruce D. The tibial plateau fracture. The Toronto experience 1968-1975. Clin Orthop Relat Res 1979; (138): 94-104

[14] Krause M, Preiss A, Müller G et al. Intra-articular tibial plateau fracture characteristics according to the "Ten segment classification”. Injury 2016; 47: 2551-2557

[15] Oestern H-J, Tscherne H. 148. Klassifizierung der Frakturen mit Weichteilschaden. Langenbecks Arch Chir 1982; 358: 483483

[16] Tscherne H, Oestern H]. [A new classification of soft-tissue damage in open and closed fractures (author's transl)]. Unfallheilkunde 1982; 85: 111-115

[17] Gustilo RB, Anderson JT. Prevention of infection in the treatment of one thousand and twenty-five open fractures of long bones: retrospective and prospective analyses. J Bone Joint Surg Am 1976; 58: 453-458

[18] Gustilo RB, Mendoza RM, Williams DN. Problems in the management of type III (severe) open fractures: a new classification of type III open fractures. J Trauma 1984; 24: 742-746

[19] Trampuz A, Zimmerli W. Diagnosis and treatment of implantassociated septic arthritis and osteomyelitis. Curr Infect Dis Rep 2008; 10: 394-403

[20] Sun Y, Wang H, Tang Y et al. Incidence and risk factors for surgical site infection after open reduction and internal fixation of ankle fracture: a retrospective multicenter study. Medicine (Baltimore) 2018; 97: e9901

[21] Barwick TW, Montgomery RJ. Knee arthrodesis with lengthening: experience of using Ilizarov techniques to salvage large asymmetric defects following infected peri-articular fractures. Injury 2013; 44: 1043-1048

[22] Phisitkul P, McKinley TO, Nepola JV et al. Complications of locking plate fixation in complex proximal tibia injuries. J Orthop Trauma 2007; 21: 83-91

[23] Marsh JL, Buckwalter J, Gelberman R et al. Articular fractures: does an anatomic reduction really change the result? J Bone Joint Surg Am 2002; 84: 1259-1271
[24] Xu S-G, Mao Z-G, Liu B-S et al. Evaluating the use of antibiotic prophylaxis during open reduction and internal fixation surgery in patients at low risk of surgical site infection. Injury 2015; 46: 184-188

[25] Suzuki T, Morgan S], Smith WR et al. Postoperative surgical site infection following acetabular fracture fixation. Injury 2010; 41: 396-399

[26] Ovaska MT, Mäkinen T], Madanat R et al. Risk factors for deep surgical site infection following operative treatment of ankle fractures. J Bone Joint Surg Am 2013; 95: 348-353

[27] Heppert V, Rheinwalt K, Winkler $\mathrm{H}$ et al. Infection of the proximal tibia after fractures - an avoidable complication. Eur J Orthop Surg Traumatol 1997; 7: 195-198

[28] Cattaneo R, Catagni M, Johnson EE. The treatment of infected nonunions and segmental defects of the tibia by the methods of Ilizarov. Clin Orthop Relat Res 1992; (280): 143-152

[29] Fang C, Wong T-M, Lau T-W et al. Infection after fracture osteosynthesis - Part I. J Orthop Surg (Hong Kong) 2017; 25: 2309499017692712

[30] Kheir MM, Tan TL, Ackerman CT et al. Culturing periprosthetic joint infection: number of samples, growth duration, and organisms. J Arthroplasty 2018; 33: 3531-3536.e1

[31] Fadel M, Hosny G. The Taylor spatial frame for deformity correction in the lower limbs. Int Orthop 2005; 29: 125-129

[32] Ali AM, El-Shafie M, Willett KM. Failure of fixation of tibial plateau fractures. J Orthop Trauma 2002; 16: 323-329

[33] Weigel DP, Marsh JL. High-energy fractures of the tibial plateau. Knee function after longer follow-up. J Bone Joint Surg Am 2002; 84: 1541-1551

[34] Kerkhoffs GM, Rademakers MV, Altena M et al. Combined intra-articular and varus opening wedge osteotomy for lateral depression and valgus malunion of the proximal part of the tibia. Surgical technique. I Bone Joint Surg Am 2009; 91 (Suppl. 2 Pt. 1): S101-S115

[35] Frosch K-H, Krause M, Frings ] et al. [Posttraumatic deformities of the knee joint: intra-articular osteotomy after malreduction of tibial head fractures]. Unfallchirurg 2016; 119: 859-876

[36] Lee O-S, Ahn S, Lee YS. Comparison of the leg-length change between opening- and closing-wedge high tibial osteotomy: a systematic review and meta-analysis. J Knee Surg 2019; 32: 372-379

[37] Marti RK, Verhagen RA, Kerkhoffs GM et al. Proximal tibial varus osteotomy. Indications, technique, and five to twentyone-year results. J Bone Joint Surg Am 2001; 83: 164-170

[38] Weber BG, Čech O. Pseudarthrosis: Pathophysiology, Biomechanics, Therapy, Results. Bern: H. Huber; 1976

[39] DiPasquale D, Ochsner MG, Kelly AM et al. The Ilizarov method for complex fracture nonunions. J Trauma 1994; 37: 629-634

[40] Moghaddam A, Zietzschmann S, Bruckner T et al. Treatment of atrophic tibia non-unions according to "diamond concept": Results of one- and two-step treatment. Injury 2015; 46 (Suppl. 4): S39-S50

Bibliografie

DOI https://doi.org/10.1055/a-0900-5344

OP-JOURNAL 2019; 35: 319-328 @ Georg Thieme Verlag KG Stuttgart · New York ISSN 0178-1715 